\title{
Very High Frequency Oscillations (VHFO) as a Predictor of Movement Intentions
}

\author{
Sara L. Gonzalez ${ }^{1}$, Rolando Grave de Peralta ${ }^{1}$, Gregor Thut ${ }^{1,2}$, \\ José del R. Millán ${ }^{3}$, Patrice Morier ${ }^{1}$, Theodor Landis ${ }^{1}$
}

${ }^{1}$ Neurology Department,

Geneva University Hospital,

24 Rue Micheli du Crest, 1211 Geneva 14, Switzerland.

${ }^{2}$ Department of Fundamental Neuroscience,

University Medical School,

Rue Michel-Servet 1, 1211 Genève 4, Switzerland

${ }^{3}$ IDIAP Research Institute.

Rue du Simplon 4, 1920 Martigny, Switzerland.

Keywords: Neural oscillations, gamma band, neural code, binding, electrophysiology.

Acknowledgements: This work is supported by the Swiss National Science Foundation (grants 3152A0-100745 and 3200B0-105867) and by the European IST Programme FET Project FP6-003758. This paper only reflects the authors' views and funding agencies are not liable for any use that may be made of the information contained herein. 
Summary: Gamma band $(30-80 \mathrm{~Hz})$ oscillations arising in neuronal ensembles are thought to be a crucial component of the neural code. Recent studies in animals suggest a similar functional role for very high frequency oscillations (VHFO) in the range $80-200 \mathrm{~Hz}$. Since some intracerebral studies in humans link VHFO to epileptogenesis, it remains unclear if VHFO appear in the healthy human brain and if so which is their role. This study uses EEG recordings from twelve healthy volunteers, engaged in a visuo-motor reaction time task, to show that VHFO are not necessarily pathological but rather code information about upcoming movements. Oscillations within the range $(30-200 \mathrm{~Hz})$ occurring in the period between stimuli presentation and the fastest hand responses allow highly accurate $(>96 \%)$ prediction of the laterality of the responding hand in single trials. Our results suggest that VHFO belong in functional terms to the gamma band that must be considerably enlarged to better understand the role of oscillatory activity in brain functioning. This study has therefore important implications for the recording and analysis of electrophysiological data in normal subjects and patients. 


\section{Introduction}

There is mounting evidence that neural oscillations play important roles in processes such as attention, perception, motor action and conscious experience (Engel et al., 2001a; Buzsaki and Draguhn, 2004); Crone et al., 1998), and that disruption or increases of activity in various oscillatory networks may be an important factor in mediating some neurological diseases (Llinas et al., 1999). Of particular interest are the neural oscillations in the gamma frequency range related among other functions to the anticipation of behaviorally relevant events and the contextual control of cortical information (Aoki et al., 1999; Engel et al., 2001b). Recent studies in rats and cats (Chrobak and Buzsaki, 1996; Grenier et al., 2001; Siegel and Konig, 2003) report a correlation between neural oscillations above $100 \mathrm{~Hz}$ and extending up to 200 $\mathrm{Hz}$ with attentive exploration and visual processing. Intracerebral recordings in monkeys show that $600 \mathrm{~Hz}$ oscillations are modulated by somatosensory stimulation (Baker et al., 2003) and that this modulation reflects the timing of cortical spike bursts.

All these animal studies suggest that very high frequency oscillations (VHFO) play functional roles similar to those reported for the classical gamma band $(30-80 \mathrm{~Hz})$. However, VHFO seems to also play a pathological role in epileptogenesis. Several intracranial studies in patients show that neocortical seizures can begin with low-amplitude high-frequency oscillations (Allen et al., 1992; Fisher et al., 1992; Alarcon et al., 1995; Traub et al., 2001) and that highfrequency epileptiform oscillations (HFEOs) appear in the interictal (between 
seizures) period (Bragin et al., 1999; Traub et al., 2001), suggesting that HFEOs may be involved in seizure generation.

While human electrophysiology has consistently investigated the functional role of gamma band oscillations, the range of frequencies above 80 $\mathrm{Hz}$ remains largely unexplored. A few electrocorticographic studies in epileptic patients report a correlation between VHFO and cognitive functions. There is nonetheless the concern that high frequency activity might have in such cases a pathological origin. It remains therefore unclear if VHFO: 1) are a natural extension of a too narrowly defined gamma band; 2) do play a different functional role than gamma band activity (GBA), or 3) are exclusively a pathological phenomenon in humans. This study aims to clarify these aspects through the analysis of electroencephalographic data recorded from healthy volunteers.

\section{Materials and Methods}

To answer these questions we recorded scalp EEG data in a population of healthy volunteers performing a visuo-motor reaction time task requiring left or right hand responses. This is a simple experimental paradigm where modulation of GBA have been consistently reported (Averbeck and Lee, 2003; Lee, 2003) and that combines some of the behavioral situations eliciting HFO in animals. We started from the hypothesis that neural oscillations playing a functional role should: 1 ) be discriminative, i.e., consistently modified by stimuli (or behavior) and thus be capable to discriminate (discriminative power) between different classes of stimuli (responses), and 2) be predictive, i.e., 
indicate in a reliable manner over trials the type of stimuli (behavior) that is being processed (executed). Latter aspect implies being able to predict subject's behavioural state (predictive power) on trials different from the ones used to evaluate their discriminative power. We analyzed the behavioral responses of subjects using the left or the right hand in separate trials and evaluated for the range of frequencies between $0-250 \mathrm{~Hz}$ the neural oscillations with most discriminative power between both movements. We then tried to decode (predict) from these oscillations and in another set of trials whether responses were done with the left or the right hand and compared this decoding with the actual manual response. Neural oscillations were considered to represent more than a simple epiphenomena but being of functional relevance when they provided accurate decoding of hand responses.

\section{Subjects and Recording}

Twelve healthy right-handed subjects (21-27 years, 6 women) were tested. Subjects were asked to fixate a central cross whose onset also served as a warning signal, followed after 3-4 $\mathrm{s}$ by a visual stimulus flashed for $60 \mathrm{~ms}$ in random order either in the left visual field (LVF) or in the right visual field (RVF) ( $4^{\circ}$ horizontal eccentricity). Subjects were instructed to respond as fast as possible with one index finger to the visual stimuli. Left and right index finger responses were assessed in separate experimental blocks and had to be given independently of visual stimulus position (simple reaction time task), thus dissociating the manual response from visual input. Reaction times (RT) were measured using an external device (mean RT $268 \pm 40 \mathrm{~ms}$ ). Each block 
consisted of 120 trials and was preceded by a training session. The position of the head was stabilized by means of a head and chin rest and the hand of the subjects rested on the response device throughout the experiment.

The electroencephalogram (EEG) was continuously monitored at $500 \mathrm{~Hz}$ during the whole experiment from 125 scalp electrodes (Electric Geodesic Inc. system, USA). Recordings were done using a cephalic reference placed at the vertex. Off-line processing of the data consisted of 1) Transformation of the data to the common average reference, 2) rigorous rejection of trials contaminated by ocular or movement artifacts through careful visual inspection, and 3) bad channel selection and interpolation. Fourteen electrodes from the lowest circle on the electrode array, i.e., closest to neck and eyes, were excluded a posteriori because of their likeliness to pick up muscular artifacts.

To account for possible electromyographic (EMG) confound in the scalp recordings of the healthy subjects due to the finger movements, we also tested one patient AM (female, 27 years, right handed), which underwent intracranial EEG recordings for presurgical epilepsy evaluation (see (Blanke and Seeck, 2003) for a detailed description of the patient). The patient performed the same visuo-motor reaction time task as used in the healthy subjects. In the patient, EEG was recorded at $200 \mathrm{~Hz}$ from subdural electrodes covering motor cortex and parietal and temporal areas of one hemisphere. The covering of motor areas was assessed by direct electrical cortical stimulation (ECS).

The local ethical committee approved the experiments, and written informed consent was obtained in all cases. 


\section{EEG data analysis}

For the analysis, we selected a stimulus-locked time window of duration equal to the subject's fastest response. This period was chosen because it is very unlikely to be contaminated by electromyographic activity due to the finger response, a potential confound of high-frequency EEG signals, as the period precedes the actual movement onset for each single trial. Note that modulation of gamma band oscillations at the level of the motor cortex has been reported to be maximal for the period preceding the actual execution of the movement (Donoghue et al., 1998) allowing for the accurate decoding of upcoming movements directions (Mehring et al., 2003; Shenoy et al., 2003) or saccades (Pesaran et al., 2002) in monkeys. In the simple visuo-motor reaction time task employed here, subjects can prepare the forthcoming action before visual stimulus onset, i.e., in the period between the warning signal that provides information about the impending event and the imperative visual signal that prompts the motor response (Leuthold, 2004). As a consequence, motor-related activity in the analyzed period after visual stimulus onset likely reflects the decision and the cerebral command to move rather than motor preparation itself.

For each individual subject, the power spectral density (PSD) was computed for all electrodes and single trials during this window using a multitaper method with seven sleepian data tapers. All computations were done in Matlab. For the healthy subjects, the whole analysis covered the frequency range from 0 to $250 \mathrm{~Hz}$, i.e. half of the frequency sampling, while for patients it 
was limited to the 0 to $100 \mathrm{~Hz}$ range, defined by the frequency sampling set to $200 \mathrm{~Hz}$.

Discriminative Power (DP) of neural oscillations

If a certain range of neural oscillations carry relevant information about the subject's functional state (preceding the left or right hand movements in our experiment), then the distribution of PSD values should have minimal overlap between tasks. Importantly, most statistical tests can indicate highly significant differences between probability distributions that considerably overlap. Thus, to select neural oscillations producing significant differences and minimal overlap between hand responses we use here a measure termed the discriminative power (DP). The DP reflects the separation between the left and right hand responses in terms of their power spectral density (PSD) for each individual frequency. It is graded between 0 and 100 , with zero representing complete overlap between both PSD distributions (no discrimination between movements is possible) and 100 representing the perfect separation between them. The DP provides an estimate per frequency of the minimum number of trials that can be unambiguously classified as pertaining to right or left movements based on a single electrode. It should not be interpreted as a classifier by itself but rather as a feature selection method. The formal mathematical definition is given in the Appendix.

Figure 1 helps to understand the difference between statistical tests based on measures of central tendency and the DP. Different insets are shown representing the histograms of two gaussian distributions with increasing 
overlaps. The significance levels for two standard statistical tests (two tailed ttest, equal means; Wilcoxon rank sum test, distributions with equal medians) and the DP value are given for each case in the inset title. It is easily seen that both statistical tests continue to detect significant differences between both distributions despite their enormous overlap. The level of overlap is however correctly indexed by the discriminative power measure.

For each subject we computed the DP for all electrodes and frequencies over the single trials that belonged to the first block of trials. Candidate frequencies that might code movement intentions were selected from the first block of trials only because the final intention was to evaluate the predictive power (PP) of every brain rhythm as an encoder of behavior in the second, independent half of the data set. To complement our study, we did also carry out a standard statistical comparison between the two PSD distributions (left and right hand responses) based on a non-parametric test: the Wilcoxon rank sum test. The Wilcoxon test (Gibbons and Chakraborti, 1992) is a nonparametric test (independent of the distribution of the data) that tests the hypothesis that two independent samples come from distributions with equal medians. A relevant question in our experimental protocol is whether VHFO reflect true neural responses or simply appears as a consequence of electromyographic activity linked to actual finger movements. Even if experimental design, careful EEG inspection, and the selected analysis windows make it very unlikely that the results are due to electromyographic contamination, we decided to carry out additional analyses to further rule out this possibility. We applied exactly the same analysis to intracranial EEG 
recordings obtained from one patient evaluated in our presurgical epilepsy unit while performing the abovementioned visuo-motor task.

\section{Predictive Power of Neural Oscillations}

To evaluate the predictive power of neural oscillations, i.e. to know if oscillations detected as relevant over the first experimental block are stable as to allow prediction of the responding hand over the second block, we used a multivariate statistical pattern recognition method known as linear Support Vector machine (Hastie et al., 2001). Statistical pattern recognition algorithms are designed to learn and later classify multivariate data points based on statistical regularities in the data set.

Learning is based in selecting some patterns (features) over the first block of trials and giving this pattern to the classifier along with a label that identifies the responding hand. The classifier then learns a mapping between patterns of brain activity and response laterality. In our analysis we selected two different types of patterns (features) in order to compare if a single frequency was better able to code the intentions of forthcoming movements than a broad frequency band: F1) The PSD at all electrodes for the most relevant frequency as identified by the DP (a topographic map for a single frequency); and F2) The PSD at the five most discriminative electrodes (according to the DP) combining all frequencies in the range $0-250 \mathrm{~Hz}$.

The percentage of correctly decoded trials was computed using a leaveone-out cross-validation. Leave-one-out (LOO) cross-validation is a method to 
estimate the predictive accuracy of the classifier. Given $n$ trials available in a dataset, a classifier is trained on (n-1) trials, and then is tested on the trial that was left out. This process is repeated $n$ times until every trial in the dataset has been included once as a cross-validation instance. The results are averaged across the $n$ trials to estimate the classifier's prediction performance. Notably, the LOO estimate is an almost unbiased estimate of the expected generalization error (Chapelle et al., 2002).

\section{Results}

Neural Oscillations with high DP.

The maximum DP over all electrodes is plotted as a function of frequency in Figure 2. Figure 3 shows the p-values obtained from the comparisons between PSDs of left and right hand responses using the Wilcoxon rank sum test. To facilitate comparison with Figure 2, we plot in Figure 3 the 1-p values. Order of the subjects is identical for both figures. The third column of Table I shows the most discriminative frequency for each subject irrespective of the electrode where it was observed.

Both the DP plot and the table indicate that a large interindividual variability is observed in terms of the oscillations showing the best DP between both responses. The Wilcoxon test results show little interindividual variability, a result expected from the small sensitivity of the test to the PSD distributions' overlap (see above). All measures (DP and p-values) do however coincide in one aspect, i.e. oscillatory activity differentiating between hand response 
laterality is restricted to rhythms above $30 \mathrm{~Hz}$ for all subjects. Besides, the best discriminative frequencies can reach values for some subjects that are far beyond the classical definition of the gamma band $(30-80 \mathrm{~Hz})$ considered relevant in electrophysiological studies. Notably, the alpha or beta bands conventionally used in EEG-based neuroprosthetic control (Millán et al., 2004; Wolpaw and McFarland, 2004), provide little discrimination in this task. Generally, the discriminative power augmented from approximately $30 \mathrm{~Hz}$ and remained elevated for some subjects up to $220 \mathrm{~Hz}$ where a drop was observed. This general tendency was confirmed by the Wilcoxon test results.

Results for the DP in the intracranial recordings of the patient are presented in Figure 4. This patient shows a maximum of DP for frequencies above $80 \mathrm{~Hz}$ with an apparent tendency to imply frequencies higher than 100 $\mathrm{Hz}$ in encoding laterality of the upcoming movement. Intracranial recordings from this patient were performed before results of the analysis of healthy subjects were available. Thus, frequency sampling was set to $200 \mathrm{~Hz}$, which is traditional in clinical settings but precludes the analysis of oscillations above $100 \mathrm{~Hz}$. Nevertheless, this result obtained in intracranial recordings where no electromyographic contamination is possible, rules out the possibility that observed differences are due to this effect.

\section{Predictive Power (PP) of Different Neural Oscillations}

The percentage of correctly decoded trials for each subject, computed using a leave-one-out cross-validation, is presented in the last two columns of Table I for features F1 (fourth column) and F2 (fifth column). 
PP varied from perfect classification (values of $100 \%$ for four subjects) to a minimum of $75 \%$ of the trials. Mean classification over subjects reached $90 \%$ of trials when using a single best rhythm for each subject and a very high rate of $96 \%$ when using the whole frequency range. As a rule, decoding of imminent movement laterality was best when based on a broad frequency band rather than when based on the most discriminative frequency. All decoding results are well above the $50 \%$ chance level expected in a two-class classification problem.

\section{Spatial Distribution of Neural Oscillations with high DP}

Figure 5 shows the averages of the individual DP values (Figure $5 \mathrm{a}$ ) and its spatial distribution ( $5 b$ and $5 c$ ) for the intervals of frequencies surrounding the two dominant peaks. Individual DP values were transformed into z-scores before averaging. Insets $5 b$ and $5 c$ show the spatial distribution of the DP for all frequencies in the intervals. The average maps over the corresponding frequency ranges are depicted in the lowermost insets. A right and left view of the map (facing each other) is given for each case. We display the maps of the individual frequencies over the considered range to highlight the spatial stability of the scalp maps around the DP peaks.

The spatial distribution of the most discriminative electrodes for the epileptic patient data is shown in Figure 6. We split the DP spatial results for the two DP peaks at $73 \mathrm{~Hz}(6 \mathrm{c})$ and $100 \mathrm{~Hz}$ (6d). To facilitate interpretation, we have incorporated in the topmost left and right panels the electrical cortical stimulation (ECS) results for this patient. The left upper panel (6a) depicts sites where ECS elicited somato-sensations in arm or fingers and the rightmost panel 
(6b) the sites where the stimulation induced movements of the hand (light blue), face (middle blue) or eyes (dark blue). Dissimilar spatial distributions of DP were observed for the two different DP peaks but with an overlap over frontal sites partially covering the motor strip (as indicated by ECS).

\section{Discussion}

Our results indicate that the strength of neural oscillations in the $30-200$ $\mathrm{Hz}$ frequency-range computed over the period between visual stimuli onset and fastest subject response allows for the accurate prediction of upcoming hand movement laterality. It confirms therefore the existence of a close correlation between oscillations above $30 \mathrm{~Hz}$ and the coding of behavioral responses. Consequently, both classically defined gamma band oscillations and VHFO play a functional role in the healthy human brain and are not necessarily of pathological origin.

The experimental design and the analysis procedure allowed us to separate the oscillations associated with lateral manual responses from those associated with lateral visual stimulus processing. The two motor response classes were by design independent of the laterality of visual presentation, as ipsi- and contralateral visual stimuli were equally distributed and thus confounded within each analyzed class. Therefore, there is no way to explain a successful prediction of upcoming hand movements based on visual processes evoked by the preceding visual stimuli. Consequently, it is most probable that the high gamma band oscillation effects observed here are linked to motorrelated processes. 
The spatial distribution of the most discriminative electrodes for the case of healthy subjects suggests a difference between the map associated to the classical gamma band peak (centered at $45 \mathrm{~Hz}$ ) and the VHFO peak. Most discriminative electrodes for the $45 \mathrm{~Hz}$ peak cluster at occipital and frontal areas while the VHFO peak is restricted to frontal electrodes with a clear lateralization to the right hemisphere. This topographical difference might be the reflection of different functional roles for classical gamma band oscillations and VHFO. While classical gamma band might underlie visuo-motor integration processes, the VHFO might reflect the later stages of motor processing including the decision to move. Nonetheless, neither the individual DP plots (Figure 2) nor the Wilcoxon test results (Figure 3 ) or the classification results (Table I) provide additional support for the hypothesis of a different functional role of gamma and VHFO. They however point into the direction of a too narrowly defined gamma band and a large interindividual variability. The existence of such large interindividual variability remains hidden when the analysis is based on standard parametric or non-parametric tests based on measures of central tendency. The reasons for such a large variability between individuals in terms of the most discriminative frequencies are not clear and will require more specific studies. It is however intriguing to observe that the (three) fastest subjects show the best discrimination for very fast oscillations (see Table I). We were however not able to detect a consistent relationship between mean reaction times and most discriminative oscillations.

Probably more significant than the large interindividual variability is the observation that half of the subjects of our study show the best decoding in the 
VHFO-range which is far above the one considered relevant for human electrophysiology. In contrast to animal studies, human electrophysiology systematically dismisses oscillations above $80 \mathrm{~Hz}$. This may obey to the fact that oscillations with frequencies above $\approx 30 \mathrm{~Hz}$ are relatively low in amplitude and are obscured by lower frequency activity. Additionally, some commercial EEG systems employ low-pass filters with cut-off frequencies from 70 to $100 \mathrm{~Hz}$. Both facts explain why high-frequency oscillations are under-recognized in human EEG studies and their functional role largely ignored. This simple methodological problem might explain why relating gamma band oscillations to behavior has remained elusive and lacks reproducibility between studies and laboratories.

When interpreting the obtained results it is important to keep in mind that EEG spectral analysis depends upon the reference (Nunez et al., 1997). The results described in this study were obtained after transforming the data to the common average reference. While there are alternatives to transform the EEG data into reference independent measures of neural activity, e.g. the Laplacian (Le et al., 1994; Babiloni et al., 1996), we preferred not to use such transformation. First, the laplacian (as other derivatives) acts as an amplifier of high (spatial) frequency noise, which is a serious concern for the inherently noisy single trial data analyzed here. Second, such transformation will considerably complicate a direct comparison of our results with animal local field potential (LFP) recordings or intracranial recordings. Nevertheless, there is no reason to expect that high frequency oscillations will be more affected by our reference selection than classical gamma oscillations. For this reason we 
believe that the main conclusion of this paper, namely that VHFO have a functional relevance, holds no matter what reference electrode were selected.

One question that derives from our results is whether VHFO about 100 $\mathrm{Hz}$ may reflect a general encoding mechanism observed across all sensory modalities or whether VHFO are restricted to the motor system. Very recently, three studies have been published that provide converging evidences on the functional role of VHFO in humans and monkeys. One study report LFP modulations within monkeys V1 over a broad band of frequencies in the $30-240$ $\mathrm{Hz}$ frequency-range with graded visual stimulus contrast (Henrie and Shapley, 2005). The range of frequencies observed in this study and the broadband extension of the observed modulation coincides well with our findings in humans. Another study using intracranial recordings over premotor cortex in one epileptic patient has reported that only the high gamma oscillations between $60-200 \mathrm{~Hz}$ were able to dissociate attention memory from motor intention (Brovelli et al., 2005). Finally, LFP recordings in the monkey motor cortex identified the range of frequencies from $80-200 \mathrm{~Hz}$ as relevant for discriminating between four possible directions of hand movements (Rickert et al., 2005). Accordingly, task related modulation of neural oscillations seems to occur over different species and sensory modalities for a broadband range of frequencies that extend from classical gamma values below $80 \mathrm{~Hz}$ up to nearly $200 \mathrm{~Hz}$. Such modulation is observable at all spatial scales using field potential measurements, i.e. LFPs, intracranial recordings and scalp EEG. 
In summary, our results have several important implications. First, electrophysiological studies in human and animals should be technically adapted to make recordings and analysis of oscillatory activity above $100 \mathrm{~Hz}$ possible while avoiding aliasing. Second, the prevalent view linking gamma oscillations to $40 \mathrm{~Hz}$ activity needs to be modified to encompass the broad frequency range that seems to be of functional relevance. Third, scalp EEG recordings convey information about VHFO that is disregarded by standard analysis procedures based on averaging over trials (e.g., ERPs). Finally, neural models of brain function require adaptation to cope with the constraints imposed for such interindividual variability, enlarged frequency range and inclusion of very fast oscillations.

\section{References}

Alarcon G, Binnie CD, Elwes RD, Polkey CE (1995) Power spectrum and intracranial EEG patterns at seizure onset in partial epilepsy. Electroencephalogr Clin Neurophysiol 94:326-337.

Allen PJ, Fish DR, Smith SJ (1992) Very high-frequency rhythmic activity during SEEG suppression in frontal lobe epilepsy. Electroencephalogr Clin Neurophysiol 82:155-159.

Aoki F, Fetz EE, Shupe L, Lettich E, Ojemann GA (1999) Increased gamma-range activity in human sensorimotor cortex during performance of visuomotor tasks. Clinical Neurophysiology 110:524-537.

Averbeck BB, Lee D (2003) Neural Noise and Movement-Related Codes in the Macaque Supplementary Motor Area. J Neurosci 23:7630-7641.

Babiloni F, Babiloni C, Carducci F, Fattorini L, Onorati P, Urbano A (1996) Spline Laplacian estimate of EEG potentials over a realistic magnetic resonanceconstructed scalp surface model. Electroencephalogr Clin Neurophysiol 98:363373.

Baker SN, Curio G, Lemon RN (2003) EEG oscillations at $600 \mathrm{~Hz}$ are macroscopic markers for cortical spike bursts. J Physiol (Lond) 550:529-534.

Blanke O, Seeck M (2003) Direction of saccadic and smooth eye movements induced by electrical stimulation of the human frontal eye field: effect of orbital position. Experimental Brain Research 150:174-183. 
Bragin A, Engel J, Jr., Wilson CL, Fried I, Mathern GW (1999) Hippocampal and entorhinal cortex high-frequency oscillations $(100--500 \mathrm{~Hz})$ in human epileptic brain and in kainic acid--treated rats with chronic seizures. Epilepsia 40:127137.

Brovelli A, Lachaux J-P, Kahane P, Boussaoud D (2005) High gamma frequency oscillatory activity dissociates attention from intention in the human premotor cortex. NeuroImage 28:154-164.

Buzsaki G, Draguhn A (2004) Neuronal Oscillations in Cortical Networks. Science 304:1926-1929.

Chapelle O, Vapnik V, Bousquet O, Mukherjee S (2002) Choosing Multiple Parameters for Support Vector Machines. Machine Learning 46:131-159.

Chrobak JJ, Buzsaki G (1996) High-Frequency Oscillations in the Output Networks of the Hippocampal-Entorhinal Axis of the Freely Behaving Rat. J Neurosci 16:3056-3066.

Crone NE, Miglioretti DL, Gordon B, Sieracki JM, Wilson MT, Uematsu S, Lesser RP (1998) Functional mapping of human sensorimotor cortex with electrocorticographic spectral analysis. I. Alpha and beta event-related desynchronization. Brain 121 ( Pt 12):2271-2299.

Donoghue JP, Sanes JN, Hatsopoulos NG, Gaal G (1998) Neural discharge and local field potential oscillations in primate motor cortex during voluntary movements. J Neurophysiol 79:159-173.

Engel AK, Fries P, Singer W (2001a) Dynamic predictions: oscillations and synchrony in top-down processing. 2:704-716.

Engel AK, Fries P, Singer W (2001b) Dynamic predictions: oscillations and synchrony in top-down processing. Nat Rev Neurosci 2:704-716.

Fisher RS, Webber WR, Lesser RP, Arroyo S, Uematsu S (1992) High-frequency EEG activity at the start of seizures. J Clin Neurophysiol 9:441-448.

Gibbons JD, Chakraborti S (1992) Nonparametric Statistical Inference, Third edition Edition. New York: Marcel Dekker.

Grenier F, Timofeev I, Steriade M (2001) Focal Synchronization of Ripples (80-200 $\mathrm{Hz}$ ) in Neocortex and Their Neuronal Correlates. J Neurophysiol 86:1884-1898.

Hastie T, Tibshirani R, Friedman JH (2001) The elements of statistical learning : data mining, inference, and prediction. New York: Springer.

Henrie JA, Shapley R (2005) LFP power spectra in V1 cortex: the graded effect of stimulus contrast. J Neurophysiol 94:479-490.

Le J, Menon V, Gevins A (1994) Local estimate of surface Laplacian derivation on a realistically shaped scalp surface and its performance on noisy data. Electroencephalogr Clin Neurophysiol 92:433-441.

Lee D (2003) Coherent Oscillations in Neuronal Activity of the Supplementary Motor Area during a Visuomotor Task. J Neurosci 23:6798-6809.

Leuthold H, Sommer, W., \& Ulrich, R. (2004) Preparing for action: Inferences from CNV and LRP. Journal of Psychophysiology 18:77-88.

Llinas RR, Ribary U, Jeanmonod D, Kronberg E, Mitra PP (1999) Thalamocortical dysrhythmia: A neurological and neuropsychiatric syndrome characterized by magnetoencephalography. Proc Natl Acad Sci U S A 96:15222-15227.

Mehring C, Rickert J, Vaadia E, Cardosa de Oliveira S, Aertsen A, Rotter S (2003) Inference of hand movements from local field potentials in monkey motor cortex. Nat Neurosci 6:1253-1254. 
Millán J del R, Renkens F, Mouriño J, Gerstner W (2004) Noninvasive brain-actuated control of a mobile robot by human EEG. IEEE Trans Biomed Eng 51:10261033.

Nunez PL, Srinivasan R, Westdorp AF, Wijesinghe RS, Tucker DM, Silberstein RB, Cadusch PJ (1997) EEG coherency. I: Statistics, reference electrode, volume conduction, Laplacians, cortical imaging, and interpretation at multiple scales. Electroencephalogr Clin Neurophysiol 103:499-515.

Pesaran B, Pezaris JS, Sahani M, Mitra PP, Andersen RA (2002) Temporal structure in neuronal activity during working memory in macaque parietal cortex. Nature Neuroscience 5:805-811.

Rickert J, Oliveira SC, Vaadia E, Aertsen A, Rotter S, Mehring C (2005) Encoding of movement direction in different frequency ranges of motor cortical local field potentials. J Neurosci 25:8815-8824.

Shenoy KV, Meeker D, Cao S, Kureshi SA, Pesaran B, Buneo CA, Batista AP, Mitra PP, Burdick JW, Andersen RA (2003) Neural prosthetic control signals from plan activity. Neuroreport 14:591-596.

Siegel M, Konig P (2003) A Functional Gamma-Band Defined by Stimulus-Dependent Synchronization in Area 18 of Awake Behaving Cats. J Neurosci 23:4251-4260.

Traub RD, Whittington MA, Buhl EH, LeBeau FE, Bibbig A, Boyd S, Cross H, Baldeweg T (2001) A possible role for gap junctions in generation of very fast EEG oscillations preceding the onset of, and perhaps initiating, seizures. Epilepsia 42:153-170.

Wolpaw JR, McFarland DJ (2004) Control of a two-dimensional movement signal by a noninvasive brain-computer interface in humans. Proc Natl Acad Sci U S A 101:17849-17854. 
Table 1: Percentage of correct classifications of laterality of upcoming movements. Classification is obtained for a time window of duration equal to the subject's fastest response using spectral features for a single frequency (F1) or the best five electrodes for all frequencies (F2).

\begin{tabular}{|c|c|c|c|c|}
\hline Subject & Mean RT & Min Best Freq. & F1 (best) & F2 (all) \\
\hline S1 & $264 \mathrm{~ms}$ & 33.1 & $92.5 \%$ & $100 \%$ \\
\hline $\mathrm{S} 2$ & $299 \mathrm{~ms}$ & 42.3 & $93.2 \%$ & $95.2 \%$ \\
\hline S3 & $352 \mathrm{~ms}$ & 47.5 & $87.2 \%$ & $100 \%$ \\
\hline S4 & $236 \mathrm{~ms}$ & 178.1 & $83.7 \%$ & $93.5 \%$ \\
\hline S5 & $211 \mathrm{~ms}$ & 145.1 & $83.2 \%$ & $85.5 \%$ \\
\hline S6 & $250 \mathrm{~ms}$ & 31.5 & $86.9 \%$ & $97.5 \%$ \\
\hline S7 & $273 \mathrm{~ms}$ & 39.1 & $92.0 \%$ & $98.8 \%$ \\
\hline S8 & $214 \mathrm{~ms}$ & 94.7 & $100 \%$ & $100 \%$ \\
\hline S9 & $288 \mathrm{~ms}$ & 199 & $75.3 \%$ & $91.3 \%$ \\
\hline S10 & $274 \mathrm{~ms}$ & 185.8 & $93.9 \%$ & $97.6 \%$ \\
\hline S11 & $276 \mathrm{~ms}$ & 67.7 & $98.2 \%$ & $100 \%$ \\
\hline S12 & $266 \mathrm{~ms}$ & 111.8 & $94.7 \%$ & $94.5 \%$ \\
\hline Mean & $267 \mathrm{~ms}$ & 97.97 & $90.1 \%$ & $96.1 \%$ \\
\hline
\end{tabular}



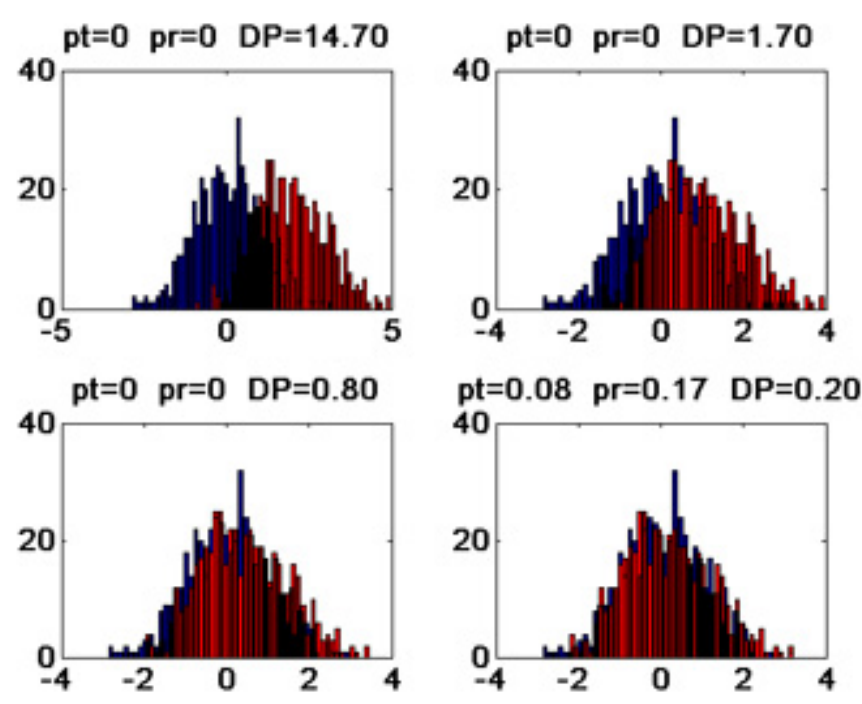

Figure 1: DP vs. Central tendency based statistical tests: Each inset represents the histograms of two gaussian distributions that differ only in their level of overlaps. Overlap increase from one inset to the next one. The title of each inset reports the significance levels for two standard statistical tests: 1) the standard t-test (pt) and 2) the Wilcoxon rank sum test (pr) equal medians). The DP values (DP) are also given in the title. Note how both statistical tests (pr and pt) indicate significant differences $(p<<0.01)$ for the distributions in the first three insets despite their huge overlap. The discriminative power measure (DP) is however sensitive to this parameter as seen from its monotonical decrease with increasing overlap. 


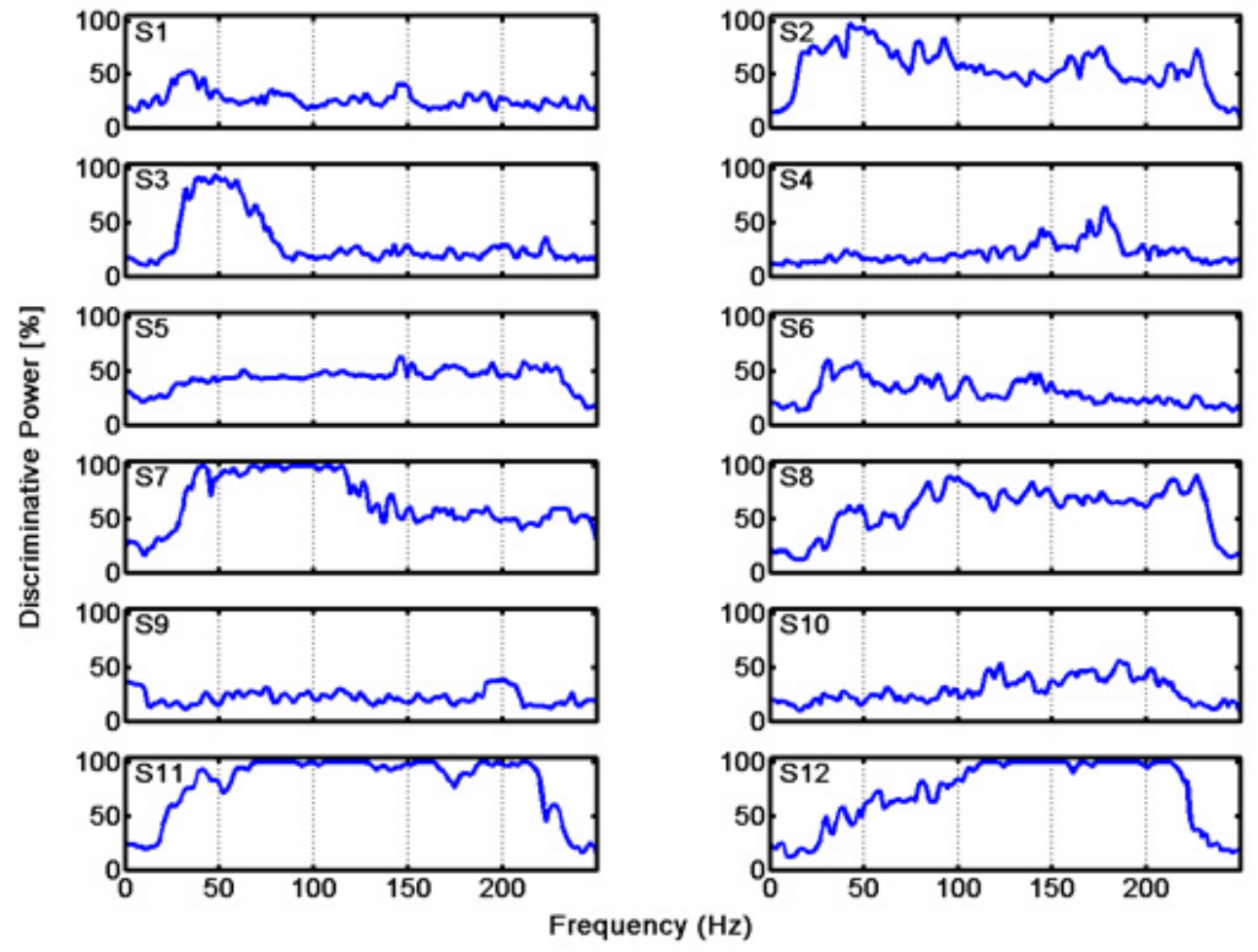

Figure 2: Discriminative Power (DP) vs Frequency (Scalp EEG recordings). Each panel represents the plot of maximum DP (Electrode providing the best discrimination between left and right hand movements) as a function of frequency for each of the 12 healthy subjects. Subjects are ordered into panels numbered left to right. Frequency oscillations above $80 \mathrm{~Hz}$ allow us to differentiate whether upcoming movements will be executed with the left or the right hand in a large proportion of subjects. 


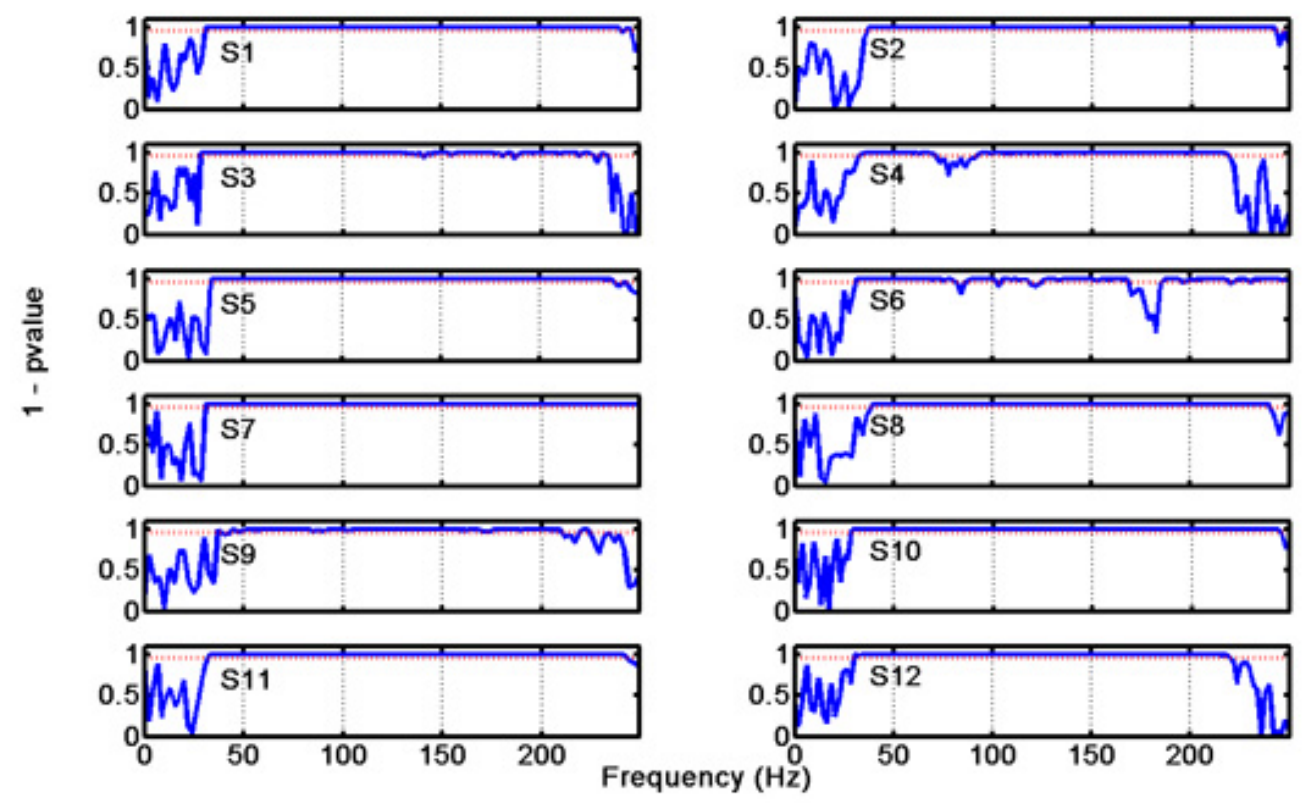

Figure 3: Oscillatory activity differing between left and right hand movements. Figure shows the 1-p-values obtained from the comparisons between left and right hand responses' PSD using the Wilcoxon rank sum test Order of the subjects is identical to Figure 2. 


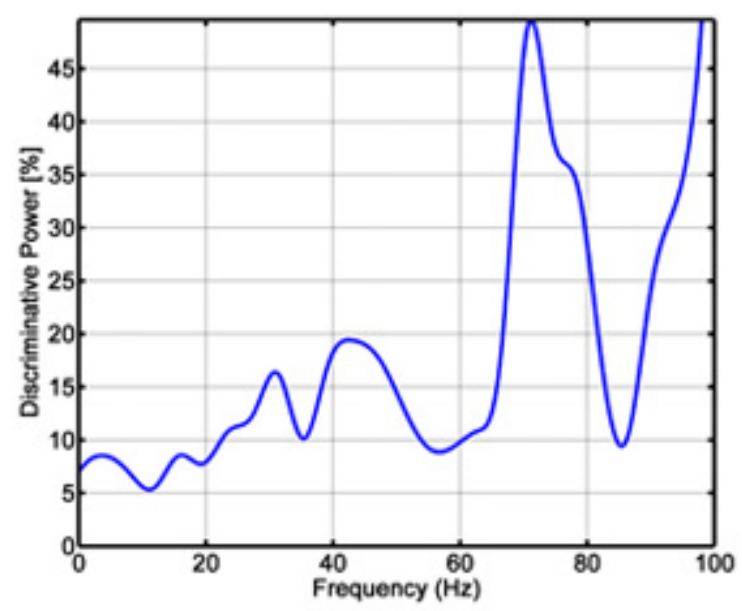

Figure 4: Discriminative Power (DP) vs Frequency (Intracranial Recordings). Plot of maximum DP as a function of frequency for the intracranial recordings in a patient. A first peak in DP is observed at $75 \mathrm{~Hz}$ with a second equally discriminative peak at $100 \mathrm{~Hz}$. Experimental filter settings (freq. sampling $200 \mathrm{~Hz}$ ) preclude analysis of oscillations above $100 \mathrm{~Hz}$. 


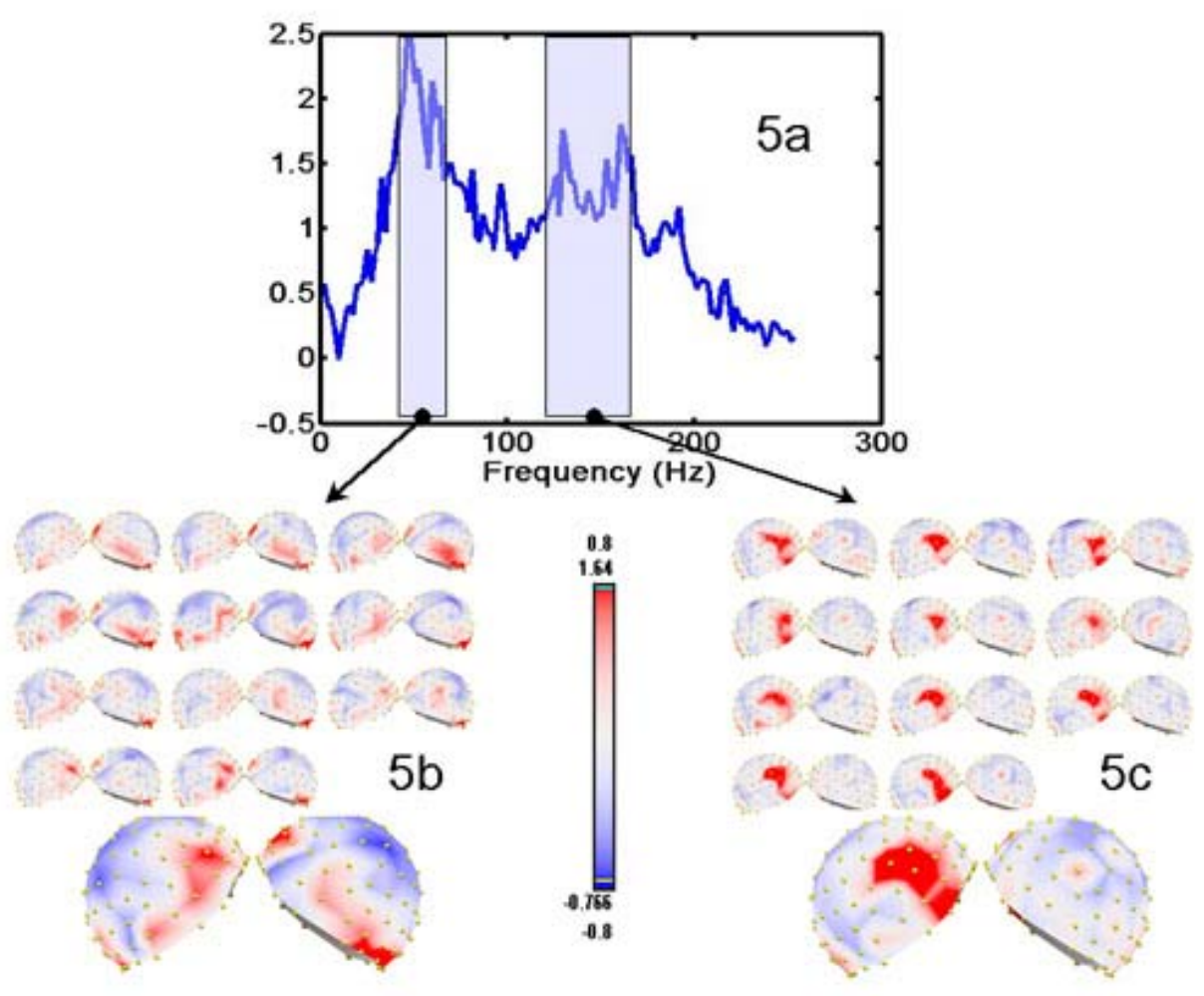

Figure 5: Spatial distributions of most discriminative electrodes. Figure $5 a$ shows the averages of the individual DP values and Figure $5 b$ and $5 c$ their spatial distribution. Two frequency intervals are shown to reflect the two dominant peaks. Small insets in $5 b$ and $5 c$ show the spatial distribution of the DP for all frequencies in the intervals. The average maps over the corresponding frequency ranges are depicted in the lowermost insets. A right and left view of the map (facing each other) is given for each case. 


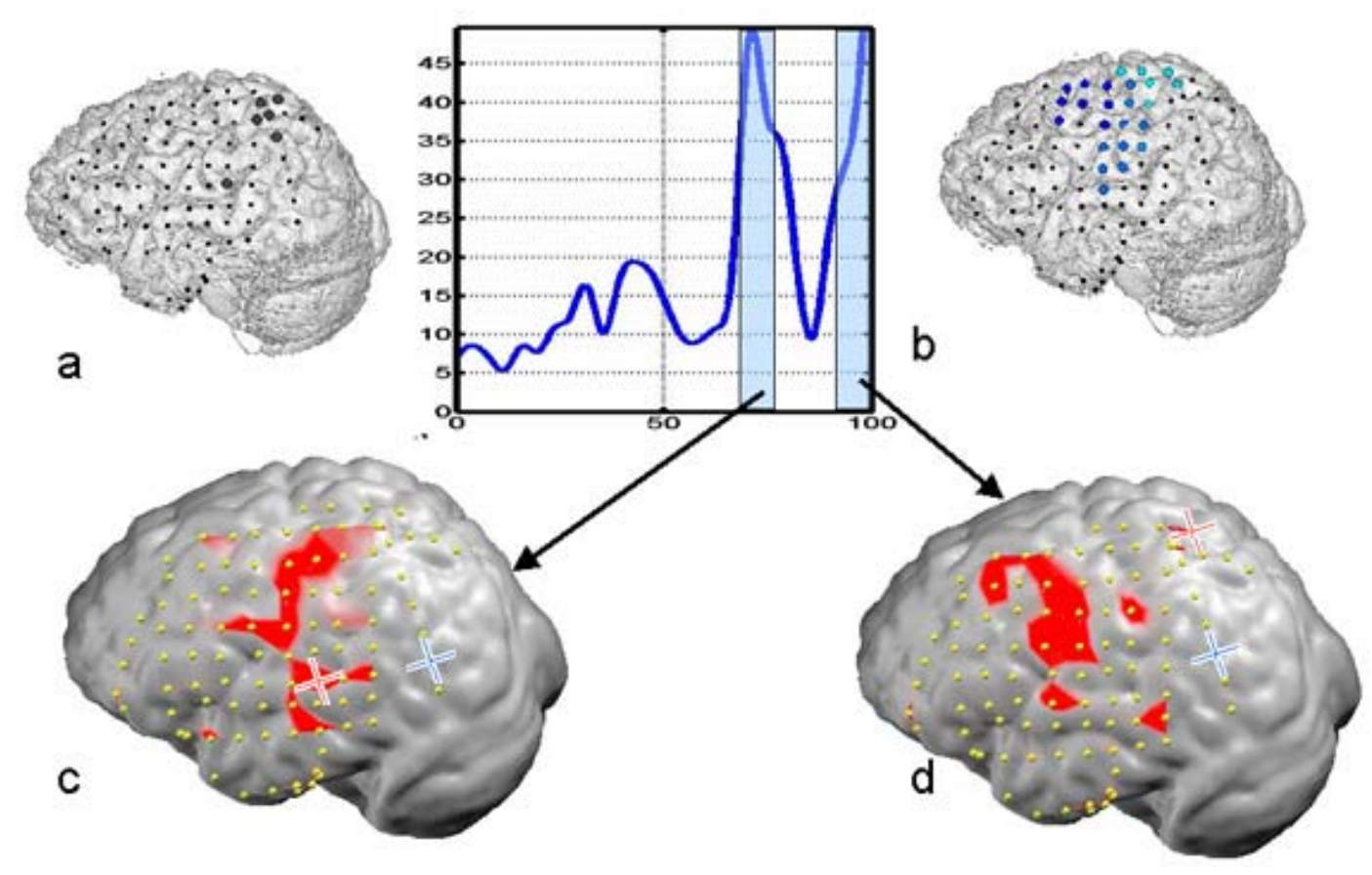

Figure 6: Spatial distribution of most discriminative electrodes for the intracranial recordings. Upper panels show the electrical cortical stimulation results for this patient. Panel $6 a$ depicts sites where somato-sensations in the contralateral arm or fingers were elicited by electrical cortical stimulation. Panel $6 \mathrm{~b}$ shows the sites where the stimulation induced motor responses of the hand (light blue), face (middle blue) or eyes (dark blue). Lower panels show the most discriminative intracranial sensors for the two DP peaks at $73 \mathrm{~Hz}(6 \mathrm{c})$ and 100 $\mathrm{Hz}(6 \mathrm{~d})$. 


\section{Appendix:}

\section{Discriminative Power Definition}

Assume that we have observed/measured or computed one variable in two different conditions $\mathrm{A}$ and $\mathrm{B}$. Denote with $\mathrm{a}(b)$ the vector containing the values corresponding to condition $A(B)$. Without loss of generality, we can assume that:

$\operatorname{amin}=\{\min$ of $a\}<=b \min =\{\min$ of $b\}$

otherwise we swap vectors $a$ and $b$.

Then the capacity of this variable to distinguish the two conditions is defined as:

$D P=0$ if $\operatorname{bmax}=\max \{b\}<=a \max =\{\max$ of $a\}$

otherwise

$$
D P=\frac{\operatorname{card}\{a<b \min \}+\operatorname{card}\{b>a \max \}}{\operatorname{card}\{a\}+\operatorname{card}\{b\}} * 100
$$

where card\{.\} stands for the number of elements in a set.

In our case, a denotes the vector formed by the PSD over all trials for a single frequency in condition $A$, and $b$ denotes the equivalent vector for the second condition. The discriminative power denotes then the percentage of times that the conditions $A$ and $B$ will be correctly identified using as a separator the lines at the minimum value of PSD for class $b$ and the maximum for class $a$. All the values lower than bmin obviously belong to class A. Similarly all values greater than amax belongs to class $B$. If the two min or max values coincide then obviously one class contains the other. 
Note that the DP is based on the extreme observed values and thus sensitive to outliers. The use of statistics based on extreme values is common in nonparametric methods as the Kolmogorov-Smirnov test or the Tuckey-Duckworth test. In similarity to non-parametric methods, the DP makes no explicit assumptions about the distribution of the data. However, this is a measure designed to evaluate finite distribution of neurophysiologically meaningful spectral data that are normally of compact support. 